\title{
Oscillations in Aggregation-Shattering Processes
}

\author{
S. A. Matveev, ${ }^{1,2,3}$ P. L. Krapivsky, ${ }^{4}$ A. P. Smirnov, ${ }^{2,3}$ E. E. Tyrtyshnikov, ${ }^{2,3}$ and N. V. Brilliantov ${ }^{5}$ \\ ${ }^{1}$ Skolkovo Institute of Science and Technology, 143026 Moscow, Russia \\ ${ }^{2}$ Faculty of Computational Mathematics and Cybernetics, Lomonosov MSU, 119991 Moscow, Russia \\ ${ }^{3}$ Institute of Numerical Mathematics RAS, 119333 Moscow, Russia \\ ${ }^{4}$ Department of Physics, Boston University, Boston, Massachusetts 02215, USA \\ ${ }^{5}$ Department of Mathematics, University of Leicester, Leicester LE1 7RH, United Kingdom \\ (Received 7 August 2017; revised manuscript received 14 October 2017; published 28 December 2017)
}

\begin{abstract}
We observe never-ending oscillations in systems undergoing collision-controlled aggregation and shattering. Specifically, we investigate aggregation-shattering processes with aggregation kernels $K_{i, j}=$ $(i / j)^{a}+(j / i)^{a}$ and shattering kernels $F_{i, j}=\lambda K_{i, j}$, where $i$ and $j$ are cluster sizes, and parameter $\lambda$ quantifies the strength of shattering. When $0 \leq a<1 / 2$, there are no oscillations, and the system monotonically approaches a steady state for all values of $\lambda$; in this region, we obtain an analytical solution for the stationary cluster size distribution. Numerical solutions of the rate equations show that oscillations emerge in the $1 / 2<a \leq 1$ range. When $\lambda$ is sufficiently large, oscillations decay and eventually disappear, while for $\lambda<\lambda_{c}(a)$, oscillations apparently persist forever. Thus, never-ending oscillations can arise in closed aggregation-shattering processes without sinks and sources of particles.
\end{abstract}

DOI: 10.1103/PhysRevLett.119.260601

Two complementary processes, aggregation and fragmentation [1-7], occur in numerous systems that dramatically differ in their spatial and temporal scales. Reversible polymerization in solutions [1,2] and merging of prions (cell proteins) [8] are typical examples on the molecular scale. On somewhat larger scales, airborne particles perform Brownian motion in atmosphere and coalesce, giving rise to smog droplets [9]. Aggregation of users on the Internet leads to the emergence of communities and forums $[3,10]$, which can further merge or split. Vortexes in a fluid flow merge and decompose forming turbulent cascades [11]. On much larger scales, aggregation-fragmentation processes take place in planetary rings, like Saturn rings, where the particle size distribution is determined by a subtle balance between aggregation and fragmentation of the rings' particles [12-16].

In spatially homogeneous well-mixed systems, the aggregation and fragmentation processes are described by an infinite set of nonlinear ordinary differential equations (ODEs) for the cluster densities. Such equations are intractable, apart from a few special cases. Still, the longtime behavior is occasionally known - the processes of aggregation and fragmentation act in opposite directions, and the cluster size distribution often becomes stationary in the long-time limit. (In exceptional cases, the typical cluster size may diverge, or the system may undergo a nonequilibrium phase transition [17-23].)

The emergence of the stationary cluster size distribution can be mathematically interpreted as the manifestation of the fixed point of the differential equations [24]. For a single ODE, fixed points determine the long-time behavior; for two coupled ODEs, fixed points and limit cycles potentially influence the asymptotic behavior. In the case of infinitely many coupled ODEs, limit cycles are feasible, yet they have not been observed in aggregationfragmentation processes. More precisely, there were signs of oscillations in a few open systems usually driven by a constant source of monomers and by a sink of large clusters. Here we report oscillations in a closed aggregationfragmentation system with strict mass conservation.

In the most important case of binary aggregation, a collision between two clusters composed of $i$ and $j$ monomers may result in the formation of a joint aggregate of $i+j$ monomers. Symbolically, $[i]+[j] \stackrel{K_{i, j}}{\rightarrow}[i+j]$, where $K_{i j}$ is the merging rate (see Fig. 1). Let $n_{k}$ be the concentration of clusters that contain $k$ monomers. These densities obey the Smoluchowski equations $[3,4]$ :

$$
\frac{d n_{k}}{d t}=\frac{1}{2} \sum_{i+j=k} K_{i, j} n_{i} n_{j}-n_{k} \sum_{i=1}^{\infty} K_{i, k} n_{i}
$$

The gain terms on the right-hand side describe the formation rate of $k$-mers from smaller clusters, while the loss terms account for the disappearance of $k$-mers due to collisions with other clusters.

In this Letter, we consider collision-controlled fragmentation which has been studied from the general perspective $[25,26]$, and it was also applied to modeling interstellar dust (a)

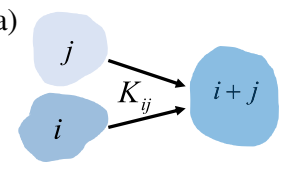

(b)

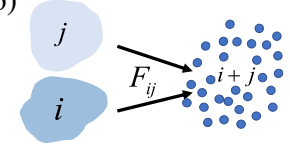

FIG. 1. Aggregation (a) and shattering (b) of clusters. 
clouds and planetary rings $[12,13,15]$. We explore the extreme version, namely, a complete shattering of two colliding partners into monomers (see Fig. 1). Symbolically, $[i]+[j] \stackrel{F_{i, j}}{\rightarrow} \underbrace{11]+[1]+\ldots[1]}_{i+j}$, where $F_{i, j}$ quantifies the shattering rate. It has been shown [12] that this shattering model is rather generic-realistic impact models with a strong dominance of small debris yield the same qualitative results for the cluster size distribution. Following Ref. [12], we assume that the shattering and aggregation kernels are proportional,

$$
F_{i, j}=\lambda K_{i, j} .
$$

The parameter $\lambda$ characterizes the relative frequency of collisions leading to merging and shattering.

Incorporating the shattering process with the shattering kernel (2) into Eq. (1), we arrive at

$$
\begin{aligned}
\frac{d n_{1}}{d t}= & -n_{1} \sum_{i=1}^{\infty} K_{1, i} n_{i}+\frac{\lambda}{2} \sum_{i=2}^{\infty} \sum_{j=2}^{\infty}(i+j) K_{i, j} n_{i} n_{j} \\
& +\lambda n_{1} \sum_{j=2}^{\infty} j K_{1, j} n_{j}, \\
\frac{d n_{k}}{d t}= & \frac{1}{2} \sum_{i=1}^{k-1} K_{i, k-i} n_{i} n_{k-i}-(1+\lambda) n_{k} \sum_{i=1}^{\infty} K_{k, i} n_{i} .
\end{aligned}
$$

Shattering leads to the increase of monomers explaining the gain terms in the first Eq. (3) and to the decrease of the densities of other clusters explaining the loss term in the second equation (valid when $k \geq 2$ ).

The kernels are symmetric $K_{i, j}=K_{j, i}$ and usually homogeneous functions of $i$ and $j$. Aggregation-shattering equations (3) for the generalized product kernels $K_{i, j}=$ $(i j)^{\mu}$ have been investigated in Ref. [12]. A more general family of kernels $K_{i, j}=i^{\nu} j^{\mu}+i^{\mu} j^{\nu}$ is often used in studies of aggregation; see, e.g., Refs. [4,27]. Here we shall focus on a special case of $\mu=-\nu$,

$$
K_{i, j}=(i / j)^{a}+(j / i)^{a}
$$

known as a generalized Brownian kernel [28]; such kernels often arise in applications $[4,29,30]$.

Below, we consider kernels (4) with $a \leq 1$; when $a>1$, the aggregation equations become ill-defined due to instantaneous gelation [31-36]. Time-dependent analytical solutions of Eq. (3) have been found [12] only for the simplest case of a constant kernel $(a=0)$.

The steady-state solutions have been obtained for a wider class of models, including an irreversible aggregation model with a monomer source [27], an aggregationfragmentation model with the generalized product kernel [12], and for a somewhat similar open system with a source of monomers and collisional evaporation of clusters with the kernel $K_{i, j}=i^{\nu} j^{\mu}+i^{\mu} j^{\nu}$ [37]. An open aggregating system with the same coagulation kernel driven by the input of monomers and supplemented with the removal of large clusters has been studied in Ref. [38]. Stable oscillations have been numerically observed [38] in this system with a finite number of cluster species. For a closed system consisting of monomers, dimers, trimers, and excited monomers, stable oscillations of concentrations have been reported [39]. Steady chemical oscillations have been also found in a simple dimerization model as well in open catalytic systems with an input of mass (see, e.g., Refs. [40,41] and references therein).

Here we consider closed systems undergoing aggregation and shattering processes described by Eqs. (3) and (4). One expects that in the closed system with two opposite processes and without sinks and sources, a steady state is achieved. This is indeed the case when $a<1 / 2$. Surprisingly, for $1 / 2<a \leq 1$ and small values of $\lambda$, a steady state is not reached and instead cluster concentrations undergo never-ending oscillations.

An important advantage of the kernel (4) is the possibility to apply highly efficient numerical methods developed in recent studies [42-47]. In simulations, we used up to $N_{\text {eq }}=2^{19}$ equations to guarantee the requested accuracy; see the Supplemental Material [48] for details.

We confirm the efficiency and accuracy of the above fast-integration method for constant kernels $(a=0)$ and find that the smaller the parameter $\lambda$, the longer it takes for the system to reach the steady state; see the Supplemental Material. This tendency persists for the kernels (4) with $a>0$. We also observe that for $a<1 / 2$, the system arrives at its steady state with a monotonic evolution of the concentrations $n_{k}(t)$. Moreover, the steady-state distribution of the cluster concentrations agrees fairly well with analytical results for $n_{k}$ derived below; this is illustrated in Fig. 2. In the language of dynamical systems [24], the system possesses a stable fixed point with steady-state cluster size distribution.

For $1 / 2<a \leq 1$, we also observed the relaxation to a steady state for sufficiently large $\lambda$; the relaxation occurs through oscillations for smaller $\lambda$, and when $\lambda<\lambda_{c}(a)$ the oscillations persist. We detected oscillations independent of the initial conditions.

Equations (3) are invariant with respect to rescaling of the conserved mass density $M=\sum_{k \geq 1} k n_{k}$ (see the Supplemental Material). Below, we report simulation results for the stepwise initial distribution

$$
n_{k}(0)= \begin{cases}0.1 & k=1, \ldots, 10 \\ 0 & k>10\end{cases}
$$

and we also simulated the evolution starting with monodisperse initial condition $n_{k}(0)=M \delta_{1, k}$ with the same mass $M=5.5$. Unless explicitly stated, the results below correspond to the initial condition (5). 

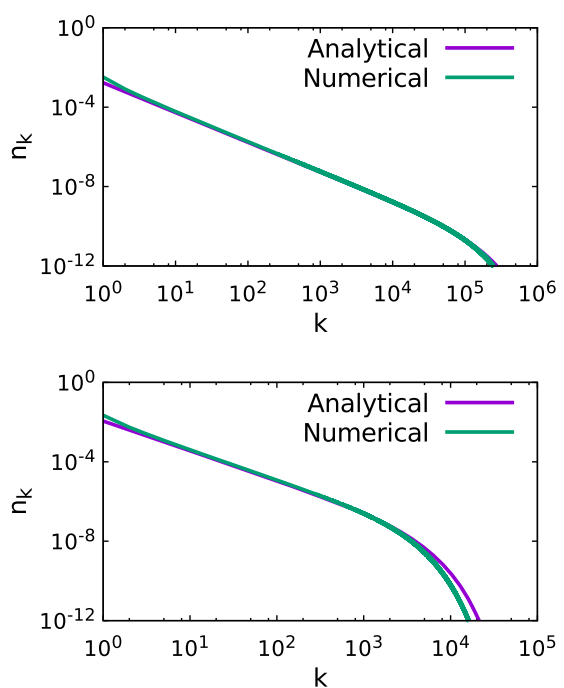

FIG. 2. Comparison of the steady-state numerical solution of Eq. (3) for the kernel (4) with $a=0.05, \lambda=0.003$ (top) and $a=0.1, \lambda=0.02$ (bottom) with the analytical steady-state solution Eq. (8).

Figures 3 and 4 depict the time dependence of the cluster density $N(t)=\sum_{k \geq 1} n_{k}(t)$. Figure 3 shows that in the range $0.6 \leq a \leq 0.8$ and $0.001 \leq \lambda \leq 0.01$, the oscillations become more pronounced when $a$ increases and $\lambda$ decreases. In Fig. 4, we show oscillating solutions of $N(t)$ in the parameter range $0.9<a \leq 1$. The new feature is the emergence of stationary oscillations. All cluster concentrations $n_{k}(t)$ perform these stable oscillations; the form of the oscillations depends on the cluster size, and the amplitude decreases with the increasing size; see Fig. 5. Figure 6 demonstrates that the system reaches a limit
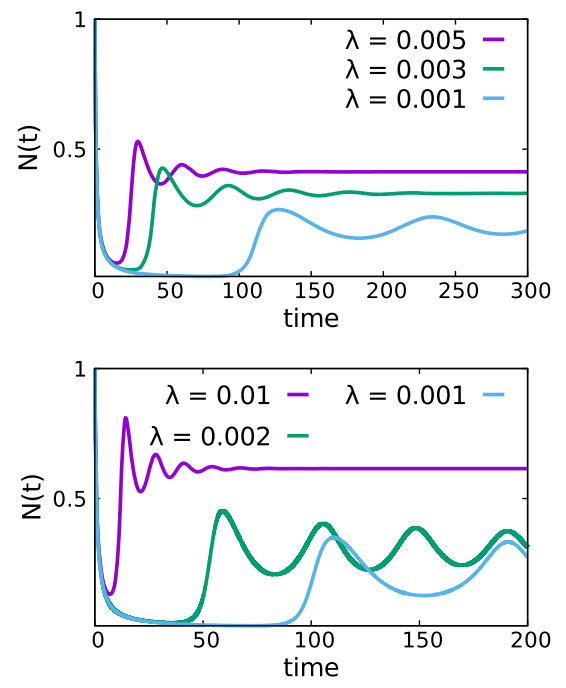

FIG. 3. Time dependence of the cluster density $N(t)$ for $a=0.7$ (top) and $a=0.75$ (bottom) and different $\lambda$. For all these systems, damped oscillations are found that tend to a steady state. The oscillations become more pronounced with increasing $a$ and decreasing $\lambda$. cycle [49], which does not depend on the total mass or initial conditions.

Oscillations in closed systems qualitatively differ from the oscillations in open systems [38]. In the closed system, the concentration oscillations occur in a form of "running waves" propagating through the cluster size distribution with some average slope (see the Supplemental Material for details); such behavior results from the conservation of the total mass yielding a limit cycle. In the open systems, the total mass is not conserved, and the pulsations of the total mass are the chief driving mechanism; the cluster mass distribution evolves in an approximately self-similar manner during each mass pulse [38].

Our results indicate the existence of a critical value of $\lambda_{c}(a)$, such that for $\lambda<\lambda_{c}(a)$, the steady-state solution is no longer stable, and instead the system approaches a limit cycle. Although for $a<0.9$ we have observed only damped oscillations, we believe that stationary oscillations would emerge for all $a>1 / 2$, but the required values of $\lambda$ are too small. The number of equations $N_{\text {eq }}$ needed for very small $\lambda$ is beyond the present computational facilities (see the Supplemental Material).

Our major observations may be summarized as follows: (1) When $a<1 / 2$, there exists a single stable fixed point for all values of $\lambda$; the steady-state distribution of cluster sizes $n_{k}$ corresponds to this fixed point. (2) When $1 / 2<$ $a \leq 1$ and $\lambda>\lambda_{c}(a)$, the system has a single stable fixed point; it may be a stable focus, resulting in dumped oscillations. (3) When $1 / 2<a \leq 1$ and $\lambda<\lambda_{c}(a)$, the system has an attractive limit cycle. (4) In the $1 / 2<a \leq 1$ range, the above assertions are conjectural and require further verification.

The emerging steady oscillations in a closed system look surprising as they seemingly violate the second law of thermodynamics. There is no contradiction due to the lack of a detailed balance, which is basically the rule in aggregation-fragmentation processes [17-21,23]. A few exceptions from this rule exist $[1,3,50,51]$, but there are no oscillations in such systems.

To probe the steady-state cluster size distribution analytically, we set $d n_{1} / d t=0$ and $d n_{k} / d t=0$ in Eq. (3) and investigate the resulting infinite system of algebraic

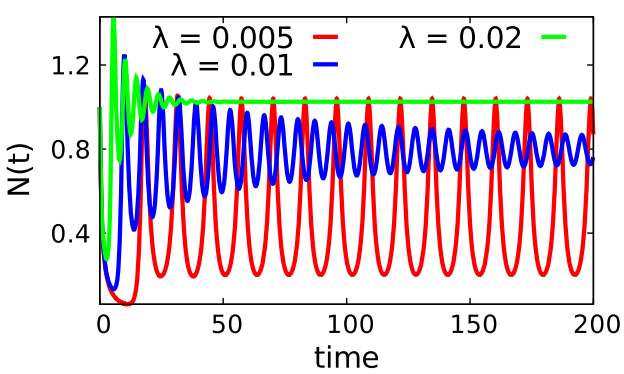

FIG. 4. Time dependence of the cluster density $N(t)$ for $a=0.9$ and different $\lambda$. For small $\lambda<\lambda_{c}(a)$, stable oscillations emerge. 

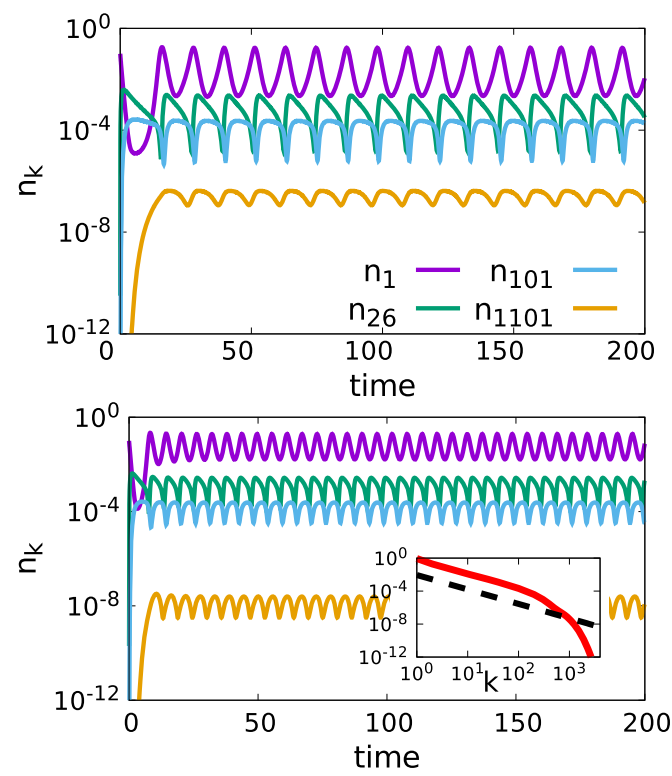

FIG. 5. Stationary oscillations of the aggregate concentrations for $a=0.95$ (top) and $a=1$ (bottom) and $\lambda<\lambda_{c}(a)$. The shape of the oscillations depends on the aggregates' size (see the Supplemental Material for details). Inset: The amplitude of the oscillations decreases with the size $k$; the dashed line is $k^{-7 / 4}$.

equations. Introducing the generating functions $\mathcal{C}_{ \pm a}(z)=$ $\sum_{k \geq 1} k^{ \pm a} n_{k} z^{k}$, we transform Eq. (3) into

$$
\begin{aligned}
& \mathcal{C}_{a}(z) \mathcal{C}_{-a}(z)-(1+\lambda)\left(M_{a} \mathcal{C}_{-a}(z)-M_{-a} \mathcal{C}_{a}(z)\right) \\
& \quad+1+\lambda) z n_{1}\left(M_{a}+M_{-a}\right)=0 .
\end{aligned}
$$

Here, $\mathcal{C}_{ \pm a}(1)=M_{ \pm a}$. To analyze $n_{k}$ for $k \gg 1$, we use the same methods as in Refs. [3,28]. When $a=0$, the tail is $n_{k} \simeq \lambda \pi^{-1 / 2} k^{-3 / 2} e^{-\lambda^{2} k}$; see Ref. [12]. This suggests that $n_{k} \simeq C k^{-\tau} e^{-\omega k}$ for $k \gg 1$, with some constants $C, \tau$, and $\omega$. Expanding the generating functions $\mathcal{C}_{ \pm a}(z)$ near $z^{\prime} \rightarrow 1-0$, where $z^{\prime}=z / z_{0}$ and $z_{0}=e^{\omega}$, we get

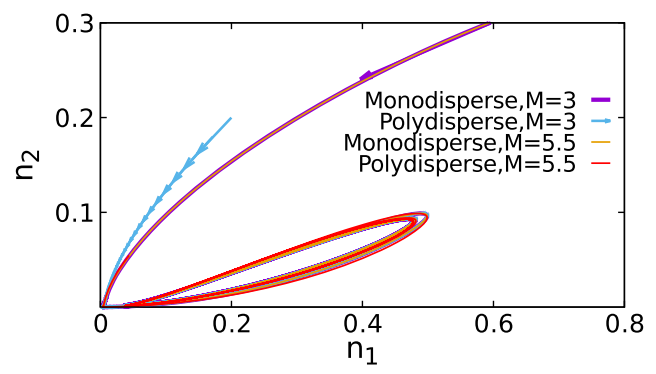

FIG. 6. Limit cycle for the steady-state oscillations in terms of $n_{1}(t)$ and $n_{2}(t)$ for $a=0.95$ and $\lambda=0.005$. For the total mass density $M=5.5$, the initial conditions are monodisperse $n_{k}=M \delta_{1, k}$ and stepwise (5). For $M=3$, the initial and current values of $n_{1}(t)$ and $n_{2}(t)$ are rescaled accordingly. The relaxation to the unique limit cycle is clearly visible.
$\mathcal{C}_{ \pm a}(z)=\mathcal{C}_{ \pm a}\left(z_{0}\right)+C \Gamma(1 \pm a-\tau)\left(1-z^{\prime}\right)^{\tau \mp a-1}$.

Here, $\Gamma(x)$ is the gamma function, and we assume that $\mathcal{C}_{a}\left(z_{0}\right)=\sum_{k \geq 1} k^{a} n_{k} z_{0}^{k}<\infty$ exists for given $a$ and $\tau$. Substituting $\mathcal{C}_{ \pm a}(z)$ into Eq. (6), we obtain terms with different powers of $\left(1-z^{\prime}\right)$. One must equate to zero each such term. In this way, we obtain equations for the zeroorder terms and the terms of the order of $\left(1-z^{\prime}\right)^{\tau \pm a-1}$, from which we find $\tau=3 / 2, \omega \simeq \lambda^{2}$, and $C=M \lambda \pi^{-1 / 2}$ (see the Supplemental Material). The final result for $n_{k}$ reads

$$
n_{k} \simeq \lambda \pi^{-1 / 2} M k^{-3 / 2} e^{-\lambda^{2} k} \text { for } k \gg 1 .
$$

The above analysis assumes that $\mathcal{C}_{a}\left(z_{0}\right)$ exists. This is consistent when $a<1 / 2$ but fails for $a \geq 1 / 2$ (see the Supplemental Material), thereby manifesting a qualitative change in the system dynamics, which we indeed observe in simulations.

To conclude, we studied numerically and analytically a system of particles undergoing collision-controlled aggregation and shattering. We considered spatially homogeneous well-mixed systems characterized by the aggregation kernel (4) and the shattering kernel $F_{i, j}=\lambda K_{i, j}$. When the parameter $a$ characterizing the kernel $K_{i, j}$ is sufficiently small $a<1 / 2$, we obtained an analytical solution for the steadystate cluster size distribution and confirmed numerically the relaxation of the size distribution to this steady-state form. For $a \geq 1 / 2$, the temporal behavior drastically depends on the shattering constant: When $\lambda>\lambda_{c}(a)$, the system relaxes to a steady state through damped oscillations, while for $\lambda<\lambda_{c}(a)$, the oscillations become stationary and persist forever (Figs. 4-6).

Using the language of dynamical systems, our observations implied that (i) for $a<1 / 2$, the governing system of ODEs possesses a single stable fixed point for all values of $\lambda$, (ii) for $1 / 2 \leq a \leq 1$, the system has a single stable fixed point (which may be a stable focus) when $\lambda \geq \lambda_{c}(a)$, and (iii) for $1 / 2<a \leq 1$ and $\lambda<\lambda_{c}(a)$, the system possesses a stable limit cycle.

Limit cycles may arise already for two coupled ODEs [24]. Still, the emergence of stable oscillations in a closed system comprising an infinite number of species and undergoing aggregating and shattering is striking. As far as we know, these oscillations have not been previously observed, and the relaxation towards the steady state was believed to be the only possible scenario.

The reported results are rather generic: For the twoparameter family of kernels $K_{i, j}=i^{\nu} j^{\mu}+i^{\mu} j^{\nu}$, we also observed never-ending oscillations when $\mu-\nu>1$ and $\lambda$ is small enough. Moreover, for the collision-controlled fragmentation models with a strong dominance of small debris, the results are similar to the reported ones for the complete shattering; see the Supplemental Material. Our findings may shed some light on the nature of the periodic formation and decay of clumps in the F Ring of Saturn-the closed 
system of particles of various masses that apparently undergo collision-controlled aggregation and fragmentation; this effect is still not fully understood (see, e.g., Ref. [52]).

This work was supported by the Russian Science Foundation, Grant No. 14-11-00806.

[1] P. J. Blatz and A. V. Tobolsky, J. Phys. Chem. 49, 77 (1945).

[2] P. J. Flory, Principles of Polymer Chemistry (Cornell University Press, Ithaca, 1953).

[3] P. L. Krapivsky, S. Redner, and E. Ben-Naim, A Kinetic View of Statistical Physics (Cambridge University Press, Cambridge, England, 2010).

[4] F. Leyvraz, Phys. Rep. 383, 95 (2003).

[5] R. D. Vigil, I. Vermeersch, and R. O. Fox, J. Colloid Interface Sci. 302, 149 (2006).

[6] J. M. Ball and J. Carr, J. Stat. Phys. 61, 203 (1990).

[7] K. Kawagoe, G. Huber, M. Pradas, M. Wilkinson, A. Pumir, and E. Ben-Naim, Phys. Rev. E 96, 012142 (2017).

[8] T. Poeschel, N. V. Brilliantov, and C. Frommel, Biophys. J. 85, 3460 (2003).

[9] R. C. Srivastava, J. Atoms. Sci. 39, 1317 (1982).

[10] S. N. Dorogovtsev and J.F.F. Mendes, Evolution of Networks: From Biological Nets to the Internet and $W W W$ (Oxford University Press, New York, 2003).

[11] V. E. Zakharov, V. S. L'vov, and G. Falkovich, Kolmogorov Spectra of Turbulence I: Wave Turbulence (Springer, New York, 2012).

[12] N. V. Brilliantov, P. L. Krapivsky, A. Bodrova, F. Spahn, H. Hayakawa, V. Stadnichuk, and J. Schmidt, Proc. Natl. Acad. Sci. U.S.A. 112, 9536 (2015).

[13] V. Stadnichuk, A. Bodrova, and N. V. Brilliantov, Int. J. Mod. Phys. B 29, 1550208 (2015).

[14] J. N. Cuzzi, J. A. Burns, S. Charnoz, R. N. Clark, J. E. Colwell, L. Dones, L. W. Esposito, G. Filacchione, R. G. French, M. M. Hedman et al., Science 327, 1470 (2010).

[15] N. V. Brilliantov, A. Bodrova, and P. L. Krapivsky, J. Stat. Mech. (2009) P06011.

[16] L. Esposito, Planetary Rings (Cambridge University Press, Cambridge, England, 2006).

[17] P. L. Krapivsky and S. Redner, Phys. Rev. E 54, 3553 (1996).

[18] S. N. Majumdar, S. Krishnamurthy, and M. Barma, Phys. Rev. Lett. 81, 3691 (1998).

[19] I. Ispolatov and P. L. Krapivsky, Phys. Rev. E 62, 5935 (2000).

[20] R. Rajesh and S. N. Majumdar, Phys. Rev. E 63, 036114 (2001).

[21] E. Ben-Naim and P. L. Krapivsky, Phys. Rev. E 77, 061132 (2008).

[22] E. Ben-Naim and P. L. Krapivsky, Phys. Rev. E 83, 061102 (2011).
[23] P. L. Krapivsky, W. Otieno, and N. V. Brilliantov, Phys. Rev. E 96, 042138 (2017).

[24] S. H. Strogatz, Nonlinear Dynamics and Chaos (Addison Wesley Publishing Company, Reading, MA, 1994).

[25] Z. Cheng and S. Redner, J. Phys. A 23, 1233 (1990).

[26] P. L. Krapivsky and E. Ben-Naim, Phys. Rev. E 68, 021102 (2003).

[27] H. Hayakawa, J. Phys. A 20, L801 (1987).

[28] P. L. Krapivsky and C. Connaughton, J. Chem. Phys. 136, 204901 (2012).

[29] M. V. Smoluchowski, Phys. Z. 17, 557 (1916).

[30] M. V. Smoluchowski, Z. Phys. Chem. 92, 129 (1917).

[31] E. M. Hendriks, M. H. Ernst, and R. M. Ziff, J. Stat. Phys. 31, 519 (1983).

[32] P. G. J. van Dongen, J. Phys. A 20, 1889 (1987).

[33] N. V. Brilliantov and P. L. Krapivsky, J. Phys. A 24, 4787 (1991).

[34] P. Laurençot, Nonlinearity 12, 229 (1999).

[35] L. Malyushkin and J. Goodman, Icarus 150, 314 (2001).

[36] R. C. Ball, C. Connaughton, T.H. M. Stein, and O. Zaboronski, Phys. Rev. E 84, 011111 (2011).

[37] C. Connaughton, A. Dutta, R. Rajesh, and O. Zaboronski, Europhys. Lett. 117, 10002 (2017).

[38] R. C. Ball, C. Connaughton, P. P. Jones, R. Rajesh, and O. Zaboronski, Phys. Rev. Lett. 109, 168304 (2012).

[39] V. I. Bykov and A. N. Gorban, Chem. Eng. Sci. 42, 1249 (1987).

[40] M. Stich, C. Blanco, and D. Hochberg, Phys. Chem. Chem. Phys. 15, 255 (2013).

[41] K. A. Fichthorn, E. Gulari, and R. M. Ziff, Chem. Eng. Sci. 44, 1403 (1989).

[42] S. A. Matveev, A. P. Smirnov, and E. E. Tyrtyshnikov, J. Comput. Phys. 282, 23 (2015).

[43] A. P. Smirnov, S. Matveev, D. Zheltkov, E. E. Tyrtyshnikov et al., Proc. Comput. Sci. 80, 2141 (2016).

[44] S. A. Matveev, D. A. Zheltkov, E. E. Tyrtyshnikov, and A. P. Smirnov, J. Comput. Phys. 316, 164 (2016).

[45] A. Chaudhury, I. Oseledets, and R. Ramachandran, Comput. Chem. Eng. 61, 234 (2014).

[46] W. Hackbusch, Computing 78, 145 (2006).

[47] W. Hackbusch, Numer. Math. 106, 627 (2007).

[48] See Supplemental Material at http://link.aps.org/ supplemental/10.1103/PhysRevLett.119.260601 for a more detailed discussion of (i) properties of the limit cycles for systems with homogeneous kernels; (ii) stationary oscillations for more general kinetic models; and (iii) derivations of analytical results.

[49] The definition of a limit cycle in the system (3) and (4) with homogeneous kernels $K_{i, j}$ and $F_{i, j}$ has some subtleties discussed in the Supplemental Material [48].

[50] D. A. Lowe and L. Thorlacius, Phys. Rev. D 51, 665 (1995).

[51] R. D. Vigil, J. Colloid Interface Sci. 336, 642 (2009).

[52] R. S. French, S. K. Hicks, M. R. Showalter, A. K. Antonsen, and D. R. Packard, Icarus 241, 200 (2014). 